\title{
DESARROLLO RURAL Y PATRIMONIO CULTURAL DE LACIANA A PARTIR DE LA PARTICIPACIÓN Y LA MEMORIA EN EL PROYECTO CAMMINUS
}

Rural Development and Cultural Heritage of Laciana from the Perspective of Participation and Memory in the Proyecto Camminus

Desenvolvimento rural e patrimônio cultural de Laciana na perspectiva da participação e da memória no proyecto camminus

\author{
Sara ÁLVAREZ GÓMEZ \\ Investigadora en patrimonio cultural. \\ sara.centroloscastros@aytovillablino.com
}

Fecha de recepción: 20/05/2020

Fecha de aceptación: 14/12/2020

RESUMEN: El monocultivo del carbón durante los últimos cien años de historia de la montañosa comarca de Laciana, León, supuso numerosos cambios en la sociedad y forjó una fuerte identidad minera. En 2020, tras el fin de la minería, este valle se encuentra en una crisis tanto económica como identitaria. En este artículo analizamos cómo recuperando la cultura inmaterial tradicional, relegada a un segundo plano durante el auge de la minería, podemos paliar diversas problemáticas que atraviesa la comarca. Presentamos el trabajo que se realiza actualmente en el proyecto camminus como parte de la fase beta de lo que algún día será el Centro Cultural de los Castros de Laciana. A partir de la participación ciudadana y la creación de un archivo lacianiego colaborativo proponemos un modelo de gestión cultural reflexivo y crítico. 
SARA ÁLVAREZ GÓMEZ

DESARROLLO RURAL Y PATRIMONIO CULTURAL DE LACIANA A PARTIR DE LA PARTICIPACIÓN Y LA MEMORIA...

Palabras clave: Identidad; patrimonio cultural; minería; desarrollo rural; participación; memoria.

ABSTRACT: Coal monoculture over the last 100 years in the mountainous region of Laciana, León, enforced various social changes and instilled a strong miner identity in the region. In 2020, the valley of Laciana is experiencing an economic and identity crisis after the end of coal mining. In this paper, we study how certain issues in this county can be alleviated by recovering the immaterial traditional culture, which was demoted by the rise of coal mining. We present the work which currently carried out as a part of the proyecto camminus, which will form the foundation of what will someday become the Centro Cultural de los Castros de Laciana. We propose a reflective and critical cultural management model based on citizen participation and the creation of a collaborative archive of Laciana.

Key words: identity; cultural heritage; mining; rural development; participation; memory.

RESUMO: A monocultura do carvão nos últimos cem anos na região montanhosa de Laciana, Leon, forçou várias mudanças sociais e levantou uma identidade forte aterrada na mineração. Em 2020, o vale está em crise econômica e de identidade. Neste artigo analisamos como a recuperação da cultura imaterial tradicional, que foi relegada durante a ascensão da mineração de carvão, pode aliviar os desafios neste município. Apresentamos o nosso trabalho, que na actualidade está em andamento no proyecto camminus como uma parte da fase beta do que um dia será o Centro Cultural de los Castros de Laciana. Propomos um modelo de gestão cultural crítico e reflexivo, baseado em a participação pública e a criação dum arquivo colaborativo de Laciana.

Palavras chave: identidade; patrimônio cultural; mineração; desenvolvimento rural; participação; memoria.

\section{INTRODUCCIÓN A LA COMARCA DE LACIANA}

El valle de Laciana (Tsaciana en patsuezu, lengua tradicional de la zona) se encuentra en el norte de León, haciendo frontera con Asturias. Es una tierra de montes abruptos y pequeños núcleos de población rurales asentados en las zonas llanas de la comarca declarada Reserva de la Biosfera en 2003. El clima frío y húmedo propicia una flora verde y viva con temporadas calurosas en verano que contrastan con la nieve del invierno. Gracias al cinturón de montañas que la rodean coloquialmente se le denomina el Valle. Aunque muchos pueblos de Castilla y León conservan instituciones medievales, arquitecturas antiguas, y una población que, aunque mermada, se corresponde con los antiguos catastros, Laciana no responde a este patrón. La estructura poblacional y social del territorio está configurada en base a los últimos 100 años, durante los cuales el motor del Valle 
SARA ÁLVAREZ GÓMEZ

DESARROLLO RURAL Y PATRIMONIO CULTURAL DE LACIANA A PARTIR DE LA PARTICIPACIÓN Y LA MEMORIA...

fue la industria minera. Realizaremos un breve análisis de los factores que han influido en este modelo social para poder finalmente explicar por qué el patrimonio cultural de Laciana es uno de los mayores activos de esta comarca.

\section{ENTORNO DEL VALLE}

El núcleo poblacional más grande de Laciana es Villablino, con una población inferior a los 5.000 habitantes (INE). Como es común en Castilla y León, las zonas rurales se encuentran muy aisladas y los pueblos son distantes entre sí. En el caso de Villablino, se encuentra a una hora de conducción de la ciudad más próxima (Ponferrada, seguida de León y Oviedo). Esta situación de aislamiento supone una desventaja, por ejemplo, a nivel sanitario (lo que no es intrínseco a las zonas rurales, sino una consecuencia de las políticas actuales centradas en lo urbano). Sin embargo, encontramos también grandes ventajas: el aislamiento permite preservar ciertas aspectos de la cultura rural: tradiciones, ritmo de la vida, bajo consumismo, acentos y lenguas, música... también fomenta el asociacionismo, la cooperación y el emprendimiento. Por último, supone un arraigo identitario mayor que en el caso de los territorios menos aislados.

Como ya se indicó antes, Laciana es una Reserva de la Biosfera. También se encuentra en la Red Natura 2000, Zona ZEPA especial de proyección de aves y está dentro de la zona de recuperación del Oso Pardo y el Urogallo, indicadores del gran patrimonio natural con el que cuenta el valle. Su hábitat natural influye directamente en el comportamiento social y en la cultura rural de la zona puesto que el ocio y el imaginario colectivo responden a su entorno. Es una zona profundamente rural en la que se llevan a cabo actividades agrícolas y ganaderas, muchas veces sin un fin económico, únicamente por tradición familiar y territorial.

En su paisaje encontramos más de diez poblados castreños distribuidos a lo largo y ancho del valle, de los cuales tan sólo dos están parcialmente excavados. La cronología de estos yacimientos abarca asentamientos formados en la I Edad de Hierro (muy escasos para la tipología castreña) hasta la ocupación medieval vinculada a la extracción metalífera de oro. Por lo tanto, la historia está marcada primero por los astures, grupo de pueblos prerromanos de origen celta del noroeste peninsular, y más tarde, por el Imperio Romano. Los asentamientos castreños indican la relevancia que tenía el territorio como punto estratégico del noroeste leonés (Fanjul Peraza, 2019).

Por ejemplo, en el castro de La Muela, encontramos un foso, restos de defensas, una muralla estrecha de la época astur y otra más ancha, de unos seis metros, de épocas posteriores, así como construcciones circulares de la época astur y reformas de la época romana. Hasta ahora, este excepcional patrimonio arqueológico no ha sido puesto en valor tanto como se merece. En la actualidad, gracias al doctorado del arqueólogo lacianiego Rubén Rubio, contamos con mucha información sobre los asentamientos castreños que podrá utilizarse para impulsar su puesta en valor. 
SARA ÁLVAREZ GÓMEZ

DESARROLLO RURAL Y PATRIMONIO CULTURAL DE LACIANA A PARTIR DE LA PARTICIPACIÓN Y LA MEMORIA...

\section{IDENTIDAD CULTURAL E INDUSTRIA MINERA}

En 1270 Alfonso X el Sabio concede a Laciana la Carta Puebla, obteniendo ciertos beneficios y estableciendo las bases para el asentamiento poblacional de la comarca. Durante los siguientes siete siglos la forma de vida de Laciana tiene pocas variaciones, manteniendo un tipo de economía agroganadera casi de subsistencia. El Valle funciona como nudo de comunicación entre Asturias y la meseta castellana. La actualidad social se conoce gracias al flujo de pastores trashumantes y mercaderes.

Los dos acontecimientos que marcarían el futuro de la comarca son, por un lado la creación de la Fundación Sierra y Pambley y más tarde la extracción carbonífera.

La Fundación Sierra y Pambley, muy vinculada a la Institución Libre de Enseñanza, funda en Villablino una escuela mercantil y agrícola a finales del siglo XIX (1886). Supone la alfabetización de la sociedad casi al completo y un gran impulso económico en las primeras décadas del siglo XX, puesto que la formación profesional ganadera y agrícola permite la creación de cooperativas que comercializan productos de la zona. Esto deja un fuerte legado pedagógico que se mantiene en el tiempo vinculando la comarca siempre a la innovación educativa y a la cultura.

El segundo acontecimiento que marca el devenir de esta tierra es el carbón. A finales del siglo XIX la extracción carbonífera es incipiente y casi artesanal. Con la llegada de distintos ingenieros y empresas extranjeras a principios del siglo XX comienza a forjarse una industria fuerte.

Coincidiendo con la creación de la empresa Minero Siderúrgica de Ponferrada (MSP) empieza a crecer la población del Valle, ya que se promueve la construcción del ferrocarril que une Ponferrada y Villablino, más coloquialmente conocido como Ponfeblino (1919). Esta conexión facilita el comercio del carbón y la industria minera se convierte en el motor económico de la zona. Debido a esto, la tradición ganadera pierde fuerza. Dejando de lado fuentes económicas alternativas, toda la comarca pasa a depender directamente de la minería.

Durante la primera década del siglo XX, Laciana cuenta con una población de menos de tres mil habitantes, que había fluctuado en unos trescientos censados durante las últimas décadas del siglo XIX. A medida que la industria del carbón crece, nuevos habitantes llegan de otras partes de la provincia, el país y el extranjero para trabajar en la mina. La población crece a un ritmo vertiginoso llegando a los casi 16.000 habitantes durante las décadas de los años 70, 80 y 90, cuando la minería alcanza su esplendor. Es notable, por ejemplo, el flujo de migración que se produjo desde Cabo Verde y supuso la creación de una comunidad de casi seiscientos caboverdianos viviendo en el Valle. La inmigración convierte a Laciana en una comunidad tolerante, con un alto grado de adaptación y un abanico de costumbres muy rico.

El entorno político y económico, sumado a las condiciones de aislamiento y entorno natural que antes comentamos, influye en las relaciones laborales y se 
forja una fuerte identidad sociocultural vinculada a la minería. Los mineros se organizan a través de sindicatos y se genera una gran conciencia de clase. Aunque casi la totalidad de trabajadores de las minas son hombres, las mujeres, relegadas al hogar y a los cuidados, se sienten igualmente partícipes y vinculadas al carbón, ya que su trabajo es el que permite que los hombres puedan dedicarse a la mina. Además, el riesgo físico que supone trabajar en esta industria vincula sentimentalmente a sus familias con el estilo de vida de la minería (Herrero Nogueira, 2004).

Esta conciencia de clase se refleja en acciones como la Marcha Negra de 1992, cuando más de quinientas familias mineras marchan a pie vestidos con la indumentaria habitual de la mina (mono y casco) desde Laciana hasta Madrid o los encierros en el Pozo Calderón. Ambas con el objetivo de que los trabajadores no sufran las consecuencias de las malas gestiones de los empresarios y de las políticas de Reconversión de la Minería del gobierno español de finales de la década de los 80 (González Rabanal, 2005).

Por diversos factores, entre ellos la gestión de los empresarios y las medidas ambientales, Laciana ve cómo se cierran sus minas hasta que llegado el año 2018 se clausura la última. Con el fin de la minería el Ministerio de Transición Ecológica firma el Acuerdo marco para una transición justa de la minería del carbón y el desarrollo sostenible de las comarcas mineras para el periodo 2019-2027. Sin embargo, la población afirma que estas medidas no están generado el impacto suficiente en la reconversión de la industria y se apunta una mala gestión de los fondos.

\section{CONSECUENCIAS DEL CARBÓN Y SITUACIÓN ACTUAL}

Las consecuencias de la dependencia económica y el monocultivo del carbón durante el siglo XX se observan ahora en todos los aspectos del territorio.

A nivel poblacional y siguiendo el patrón de la España Vaciada, encontramos en 2020 menos de ocho mil personas censadas en la comarca (hay que tener en cuenta que algunas de estas personas residen fuera aunque no quieren cambiar su empadronamiento). Aunque llevamos años observando como la España rural se vacía, es ahora cuando se empieza a aceptar socialmente la emergencia de esta situación. Las nuevas generaciones, a pesar de querer vivir en su pueblo natal, se ven obligadas a desplazarse a los núcleos urbanos para encontrar estabilidad laboral. Aunque la industria minera supuso un gran impulso de la economía de la zona, una consecuencia del monocultivo fue no preservar ni fomentar otras fuentes económicas (como la antigua ganadería o distintas industrias que podrían haberse asentado en el Valle). Así se desencadena la actual crisis económica, agravada por el marco de la crisis española del 2008. Esto evidencia no solo la necesidad de buscar nuevas fuentes económicas, sino de incluir el impacto económico positivo como requisito para cualquier proyecto que se ponga en marcha en la comarca. No parece coherente, por ejemplo, llevar a cabo un gran proyecto sobre 
el patrimonio inmaterial de Laciana si éste no fomenta la prosperidad económica de su población.

Por otra parte y como ya hemos indicado, durante el último siglo se forjó una fuerte identidad minera. Trajo consigo un gran sentimiento de pertenencia, colaboración y asociacionismo que se conserva como legado. Este nuevo sentimiento identitario desplazó en gran medida al conjunto de tradiciones y costumbres que lo precedían. Ahora la población de Laciana puede hacerse una pregunta: ¿si ya no somos mineros, qué somos?. Corre el peligro de que la identidad cultural venidera se construya a partir del pesimismo. La falta de cohesión social que conlleva este desarraigo de su propia identidad se ve reforzada por la, en cierta medida, decadencia de las estructuras poblacionales. Si en la comarca se edificó vivienda para casi dieciséis mil habitantes y ahora la población ha mermado hasta la mitad, los locales cerrados y los edificios vacíos ocupan gran parte de las calles. Así se refuerza un sentimiento de nostalgia por los tiempos mejores. En estos momentos la población debe definirse a sí misma, por ello es necesario que los procesos de participación y colaboración ciudadana formen parte de las administraciones y se decida el futuro de Laciana en común. Además parece fundamental que las vías de desarrollo económico no se fijen únicamente en el pasado sino que exploren metodologías innovadoras.

En cuanto al entorno natural de Laciana, la minería a cielo abierto ha sido motivo de polémica y de discusión entre grupos ecologistas y empresarios del carbón durante años. En 2011 una sentencia del Tribunal de Justicia de la Unión Europea declara que las minas a cielo abierto de Laciana no cumplen las directivas medioambientales (Pernas García, 2011). Es una de las varias sentencias y procesos judiciales que sufrieron las minas hasta que su desaparición. Miles de familias continúan viviendo gracias a las prejubilaciones que dejó la actividad minera. Pero no hay que olvidar el impacto ecológico que supuso y aún supone para un entorno natural: se talaron bosques completos y se "comieron montañas» con el objetivo de extraer carbón, el agua del Río Sil se contaminó por los metales pesados de la mina y se utilizaron parajes naturales como escombreras. Gracias al trabajo de diversos agentes y del Ayuntamiento de Villablino, en los últimos años se han catalogado y restaurado muchas de las escombreras y así paliado los efectos de estas agresiones a la naturaleza. Cabe destacar el gran patrimonio industrial y minero que se puede ver hoy en la comarca y que está aún por analizar y gestionar para su puesta en valor. Es por esto importante, que las alternativas económicas que se planteen a partir de ahora cumplan con medidas de sostenibilidad, preservación del patrimonio natural y que fomenten la economía de proximidad.

Tras este breve análisis podemos entender por qué la cultura de Laciana ha cambiado tanto en los últimos cien años. Los flujos de migración, el relevo identitario, las fuentes económicas y la política transformaron el perfil sociocultural de esta comarca. 
SARA ÁLVAREZ GÓMEZ

DESARROLLO RURAL Y PATRIMONIO CULTURAL DE LACIANA A PARTIR DE LA PARTICIPACIÓN Y LA MEMORIA...

En medio de la vorágine de la minería, la cultura tradicional fue quedando relegada, muchas veces, a la población más envejecida. Gracias al trabajo de asociaciones, vecinos, vecinas y algunas administraciones por recuperar antiguas tradiciones, es que aún hoy podemos hablar de este patrimonio inmaterial.

\section{CUlTura LACIANIEGA Y TRADICIÓN ORAL}

La situación geográfica de Laciana le da una dimensión cultural muy interesante. Por su cercanía a Galicia y Asturias pero perteneciendo a León, encontramos tradiciones parecidas en las tres provincias que resultan propias de este territorio. Se podría decir que la gran protagonista es la tradición oral, gracias a la cual se han conservado leyendas, tradiciones, remedios naturales, cantares $\mathrm{O}$ poemas. Nos encontramos en un territorio que combina las costumbres de la tradición pagana de los pueblos de origen celta con las festividades religiosas que surgieron de la conciliación por parte de la Iglesia Católica. Damos a continuación unos breves apuntes sobre la cultura lacianiega.

Las leyendas que se conservan están muy vinculadas a la magia, las criaturas mitológicas de los montes o los personajes misteriosos que aparecían repentinamente por los pueblos con remedios o maldiciones. También, como es habitual, las leyendas narran fenómenos extraños del territorio como la desaparición de ganado en ciertos lugares. La leyenda que mostramos a continuación ejemplifica esta vinculación de lo sobrenatural con la naturaleza.

La Hija de la Montaña.

Subiendo a Cuetonidio por la braña de Rabanal de Arriba, junto a la Fuente de la Nieta, se contaba un relato de pastores. Se decía que un día llegó un coloso de color negro al Valle de Laciana. Este trasgo gigante vagaba por las sendas y los bosques profanando tumbas, devorando el ganado y atacando a las jóvenes vírgenes. La Montaña, cansada de la masacre, se enfrentó al trasgo clamando justicia por el valle. El trasgo, ignorándola, la sometió y violó, huyendo después valle abajo. La hija de la Montaña, que había sido testigo de todo ello, decidió engañar al trasgo disfrazándose de virgen con flores para hacer que el coloso volviese a la cima de su madre, la Montaña. El coloso cayó en su trampa, y cuando llegó a la cima, la Montaña se abrió en dos, estremecida y loca de rabia, y lo engulló en venganza por sus actos. Cuentan que las aguas del Sil dieron así sepultura al coloso de color negro, y por ello corren hoy tan oscuras. La Montaña, sublime e invicta, quedó desde entonces como guardiana eterna de las gentes de Laciana.

Leyenda recogida por Florentino Agustín Díez en su obra Laciana (Memoria de su antiguo y patriarcal Concejo) y adaptada por proyecto camminus.

También ligado al pasado astur, se celebra el magosto o calbote (amagües$t u$ en asturias y magosto en Galicia) que consiste en encender una hoguera y asar castañas sobre las brasas para celebrar el paso al invierno. Cuenta con una gran simbología ya que se vinculan las castañas consumidas con almas liberadas. 
SARA ÁLVAREZ GÓMEZ

DESARROLLO RURAL Y PATRIMONIO CULTURAL DE LACIANA A PARTIR DE LA PARTICIPACIÓN Y LA MEMORIA...

También se conserva la tradición de la matanza durante el mes de noviembre coincidiendo con la celebración del Samartino, en la que las familias se reúnen para sacrificar al cerdo que han alimentado y del que se extrae manteca y carne que se consume durante todo el año. La gastronomía está considerada uno de los atractivos del Valle. Es una cocina muy tradicional que se ha mantenido en el tiempo, compuesta por platos enérgicos propios de la montaña. También es habitual el uso de plantas silvestres para la elaboración de remedios naturales, limpieza y cocina en el hogar.

Parte de este patrimonio inmaterial es el llamado patrimonio lingüístico que durante los últimos años ha tomado de nuevo gran importancia. El patsuezu (leonés occidental o asturleonés) se considera una lengua histórica conservada desde la Edad Media que ha llegado a nuestros tiempos gracias al aislamiento al que antes hacíamos referencia y al trabajo de asociaciones y vecinos que han pujado por su conservación. Hasta hace pocos años se consideraba lengua de analfabetos y muchas de las personas que lo hablaban lo hacían con vergüenza. Recientemente se han publicado obras en patsuezu de diversos autores como Eva González González o Guadalupe Lorenzana Rodríguez. El aprendizaje de esta lengua continua a través de los cursos que imparte el lingüista Roberto González Quevedo. Guadalupe Lorenzana afirma «Entovia güei, mia madre ya las personas más viechas dícenme qu'el castel.lanu siéntese mechor, que a el.la présta-l.ly más que fale en castel.lanu qu'en pal.luezu. Amira... palos viechos yera fala d'analfabetos ya paletos. Dába-l.lys vergoña falalu delantre la xente ya, a min pasóume outru tantu muitísimu tiempo»/ "Todavía hoy, mi madre y las personas mayores me dicen que el castellano suena mejor, que a ella le gusta más que hable en castellano que en patsuezu. Fíjate... Para los mayores era sinónimo de incultura, de paletismo. Les daba vergüenza hablarlo delante de la gente y lo mismo me pasó a mí durante mucho tiempo". (El pal.luezu, la l.lingua l.lionesa de la Muntaña Ocidental, 2017).

De la mano del patsuezu va la música tradicional, que comparte repertorio con Asturias y Galicia. Instrumentos como el pandero o las castañuelas, siempre fabricados de forma artesanal, conviven con la popularidad de la gaita y del folk más contemporáneo, estilos que se vinculan habitualmente con el mundo celta. Acompañando la música tradicional se practica el baile regional, conocimientos que se comparten en grupos y asociaciones folklóricas.

Este amplio patrimonio inmaterial se ha transmitido de generación en generación por medio de la celebración de calechos y filandones, formas tradicionales de reunión en torno al fuego en las que se cuentan leyendas e historias mientras se hace algún trabajo manual como la talla o los remiendos. Actualmente estas reuniones con muy escasas y cuando se celebran se hacen de forma más flexible sin la necesidad de hogueras ni trabajos manuales. Consideramos necesario seguir promoviendo la transmisión oral del conocimiento popular y trabajar para la recuperación de aquellas tradiciones que han perdido fuerza. 
SARA ÁLVAREZ GÓMEZ

DESARROLLO RURAL Y PATRIMONIO CULTURAL DE LACIANA A PARTIR DE LA PARTICIPACIÓN Y LA MEMORIA...

\section{PATRIMONIO CULTURAL Y PROYECTO CAMMINUS}

En los últimos años se han puesto en marcha proyectos para superar la crisis como el retorno de la actividad agroganadera, que sigue siendo una posible fuente económica pese a que no resulta fácil recuperar antiguos modelos económicos. También se ha propuesto a Laciana como destino turístico rural vinculado a las actividades en la naturaleza. Por otra parte, existen diversos proyectos de emprendimiento privado relacionados con los recursos endógenos como la producción de miel o las granjas ecológicas de gallinas ponedoras. Poco a poco van surgiendo diversos proyectos que pujan por la vida en el Valle y que respetan el hábitat en el que se desarrollan.

Desde la perspectiva de la gestión cultural, encontramos en el patrimonio cultural un recurso sostenible y un gran activo para la revitalización económica y social del Valle. Analizando nuestro patrimonio desde esta posición, nos encontramos con el patrimonio natural y el cultural, ambos relacionados, puesto que el segundo está condicionado por el primero. Siguiendo el modelo italiano, cuando nos referimos a bienes culturales o patrimonio cultural, hablamos de las distintas manifestaciones inmateriales, históricas, arqueológicas o industriales que encontramos en la comarca. Una de las formas de responder a algunas de las problemáticas que hemos planteado anteriormente, es la gestión de este patrimonio y su puesta en valor con los siguientes objetivos:

- Fomentar y difundir el patrimonio cultural de Laciana trabajando para su protección y preservación.

- Debatir sobre la identidad de Laciana señalando aspectos culturales desatendidos durante el último siglo.

- Ampliar la oferta cultural de la comarca para que suponga una alternativa real al ocio urbano.

- Fomentar el crecimiento económico de la comarca:

- Impulsar el turismo sostenible cultural, ofreciendo contenidos que dialoguen con el patrimonio natural y creen un mismo discurso sobre Laciana.

- Fomentar y participar en una red de apoyos entre distintos agentes culturales del noroeste peninsular para compartir recursos, dar visibilidad y crecer en común.

Todo estos objetivos se plantean desde el proyecto camminus. El proyecto nace a principios de 2019 para crear un innovador centro cultural sostenible basado en la cultura de Laciana. Se pretende reutilizar el Aula de Interpretación de los Castros. Este espacio se proyectó en el antiguo edificio de las escuelas de Rioscuro (pedanía de Laciana), construido a principios del siglo XX y ampliado en 2013 para albergar este aula. Sin embargo, debido a problemas económicos, nunca llegó a funcionar a pleno rendimiento y en la actualidad se encuentra casi en desuso. El proyecto camminus, impulsado por el Ayuntamiento de Villablino y 
colaborando con la Cátedra de Turismo Sostenible y Desarrollo Local de la UNED, busca apoyo y financiación pública para revivir esta infraestructura y crear el Centro Cultural de los Castros de Laciana, un centro que funcione no solo como espacio físico para albergar actividades culturales, sino como sede de un trabajo más complejo y amplio que responda a los objetivos anteriormente planteados. El proyecto sigue los principios que hemos indicado como necesarios para la comarca: sostenibilidad económica, participación ciudadana, bajo impacto en el entorno natural, economía de proximidad, colaboración entre distintos agentes e innovación. Sin profundizar en el funcionamiento que se proyecta para el centro cultural, como el sistema de difusión, los principios éticos y laborales o la innovación pedagógica, presentamos una muestra del contenido en el que se está trabajando:

- Actividades renovadas periódicamente: rutas, talleres de fin de semana, visitas matinales, campamentos y festival de verano.

- Espacio de libre acceso con mobiliario didáctico, equipo informático, biblioteca móvil y zona de descanso.

- Espacio de muestra sobre la cultura local y los Castros de Laciana.

- Colaboraciones con agentes locales y externos: participación ciudadana, fomento del tejido cultural, cesión de espacios y vinculación con instituciones universitarias.

- Vínculos con agentes del territorio que trabajen con el patrimonio natural para impulsar el turismo rural sostenible.

- Investigación, preservación y difusión del patrimonio a partir del Archivo Lacianiego.

- Residencias de artistas para la creación de dispositivos de mediación entre el público y el contenido.

- Espacio gastronómico didáctico con productos de la zona (kilómetro 0).

Nos centramos ahora en el trabajo que se está realizando actualmente en lo que llamamos Fase Beta del proyecto camminus, atravesado por dos temas centrales: el diseño del centro a través de la participación ciudadana y la creación del Archivo Lacianiego.

Aunque se proponga la estructura desde el equipo del proyecto, el contenido y las metodologías deben decidirse a través de la participación ciudadana. Es necesario que la población tome decisiones sobre un espacio cultural público que formará parte del tejido social de su comarca.

Sobra decir que la participación es algo se debe fomentar desde cualquier institución pública. Si lo público está al servicio de la población pero esta misma no tiene voz en la toma de decisiones, se crea una confrontación entre lo público y el público. Además, la participación fomenta la implicación y la confianza en la institución, necesaria en un contexto que debe reflejar la realidad de la comarca. Si los usuarios del centro sienten la confianza para dialogar con el equipo de gestión, la detección de problemas es más rápida y se pueden solucionar de forma eficaz. 
Parece ilógico gestionar un espacio en el que vive la cultura inmaterial sin tener en cuenta a su protagonista, la población. Un centro cultural debe ser un espacio habitado que se sienta como propio, no un lugar de paso sin memoria.

Se han llevado a cabo varias jornadas de participación con distintos colectivos de la población y encuestas online. Desde agosto de 2019 a enero de 2020 se llevaron a cabo unas dos jornadas mensuales. En la primera jornada, de tono festivo, se presentó el proyecto y se hizo una consulta a los participantes sobre la situación actual del Valle y las distintas actividades que les gustaría que ofertase el centro cultural. Los habitantes de Rioscuro, pueblo en el que se ubica el espacio, demandaban sobretodo actividades comunitarias como, por ejemplo, comidas populares. También se llevó a cabo un taller relacionando la música con la memoria en el Centro Sociosanitario Valle de Laciana, donde tras una muestra de música tradicional de mano del Grupo Folklórico La Farándula, se produjo una conversación sobre las tradiciones de los participantes, todos ellos en una franja de edad comprendida entre los 70 y 95 años.

En cinco ocasiones se realizó un taller sobre cultura, sobreinformación y pensamiento crítico en los institutos de la comarca con la intención de dialogar sobre la cultura local y la colaboración. En estos talleres surgió la idea de crear un grupo de acción autónomo para personas menores de veinte años, que lleven a cabo distintas actividades que ellas mismas organicen (siempre con el acompañamiento del equipo gestor para no fomentar el trabajo gratuito).

Se celebró la fiesta de Samhain, de orígenes celtas, mostrando información sobre las distintas tradiciones de los territorios del noroeste peninsular. Se contaron leyendas en forma de calecho y se consumieron castañas asadas. A su vez, los y las más pequeñas tallaron distintas hortalizas y crearon collares de castañas, mientras el resto de participantes compartían sus propias tradiciones y leyendas a través de post-its en una infografía colaborativa.

Tuvo lugar un taller para menores de doce años en el que los niños y niñas exploraron el centro y dibujaron en un gran plano del edificio qué podría ofrecer el centro. Cabe destacar las múltiples peticiones de estos niños para construir piscinas de unicornios y sillones mágicos que probablemente no se puedan llevar a cabo.

Las conclusiones, ideas, peticiones y conversaciones se registraron por medio de relatorías gráficas y posteriormente se volcaron en documentos. Toda esta información se utilizará para diseñar las actividades y darle forma al contenido. La participación ciudadana continuará siendo un aspecto troncal del funcionamiento del Centro Cultural de los Castros de Laciana.

En segundo lugar presentamos la creación de un Archivo Lacianiego. Habitualmente utilizamos el término archivo para referirnos a un conjunto de documentos con un orden establecido. El archivo es un mecanismo de preservación de la memoria y la historia de cierto grupo o ámbito y puede suponer un vínculo perpetuo con las raíces de un territorio. Es un salvavidas de la memoria colectiva de una sociedad. Los elementos de un archivo no son meros documentos ordenados, contienen decisiones, acciones, recuerdos e identidad (RAMÓN, 2018). 
SARA ÁLVAREZ GÓMEZ

DESARROLLO RURAL Y PATRIMONIO CULTURAL DE LACIANA A PARTIR DE LA PARTICIPACIÓN Y LA MEMORIA...

La creación de un archivo de Laciana supone no solo preservar la identidad de la comarca, sino construir un mecanismo para difundir el patrimonio (no solo histórico) del territorio. Tiene gran valor para la población de a pie, investigadores y académicos.

El concepto de archivo se encuentra normalmente situado en el imaginario colectivo como una sala gris llena de carpetas con infinitos documentos. Sin embargo, el archivo que se plantea desde proyecto camminus no responde a este modelo. Se pretende expandir el concepto y crear un archivo en el que tenga cabida todo y todos. El objetivo es que sea capaz de documentar y preservar cualquier elemento que se considere relevante: personajes, especies de flora y fauna, lugares, leyendas, tradiciones, fechas, representaciones sonoras y visuales, documentos, testimonios, eventos, noticias, fenómenos meteorológicos... Sería una entidad en constante cambio y revisión con el objetivo de adaptarse a la realidad pasada y presente de la comarca.

El proceso de la creación del archivo deberá ser colaborativo y horizontal. $\mathrm{Al}$ igual que la participación ciudadana, la creación comunitaria refuerza el sentimiento de pertenencia empoderando a la población, que finalmente será la que decida si un elemento merece ser archivado. El funcionamiento interno y las cuestiones prácticas serán llevadas a consenso.

Para crear este archivo se organizará, a lo largo de 2020, un grupo de archiveros divididos en grupos de trabajo según distintos ámbitos (que se decidirán en las primeras sesiones de trabajo). Avanzarán de forma autónoma pero se pondrán en común las decisiones de cada equipo. La forma de crear estos grupos de trabajo será a través de una convocatoria abierta para cualquier residente de Laciana. El desarrollo de la iniciativa se comunicará en las redes sociales de una forma abierta y transparente, a través de las cuales se podrá participar en el proyecto si así lo deciden los grupos de trabajo o añadir nuevos miembros. Una vez asentadas las bases del archivo, se diseñarán procesos para poder añadir nuevos elementos, algo que podrá hacer cualquier persona.

La intención es disponer del archivo tanto de forma física como digital, lo que también supone decidir cómo se traducirán los documentos físicos al plano digital. En cuanto al espacio físico, se buscará que además de práctico, resulte atractivo y dinámico.

Las iniciativas que surjan gracias a la existencia del archivo alimentarán el contenido del centro cultural. Si se dispone de forma ordenada de amplia información y referentes de un territorio, será más fácil organizar acciones de difusión del patrimonio. A partir de elementos del archivo se pueden organizar jornadas, actividades y conferencias sobre temas en particular. Si se decidiera que uno de los elementos archivados, por ejemplo la celebración de una boda tradicional lacianiega, es merecedor de más atención, se podría organizar una taller que al mismo tiempo serviría para alimentar el propio archivo. Además, se pueden celebrar jornadas temáticas con la presencia de personas expertas para nutrir una sección específica, como podría ser una jornada sobre el patrimonio industrial 
minero, que a su vez sería una interesante actividad para el turismo rural. A día de hoy se ha propuesto una iniciativa basada en la creación de un mapa tanto físico como virtual que señale distintos lugares o curiosidades de Laciana y pueda estar disponible para el turismo, funcionando como ventana a la sociedad lacianiega.

Por tanto, este archivo no solo contribuiría a la preservación del patrimonio y el refuerzo de la identidad cultural, sino que supondría una fuente de recursos para fomentar el turismo sostenible e impulsar la economía del Valle.

\section{REFERENCIAS BIBLIOGRÁFICAS}

Agustín Díez, F. (1946). Laciana (Memoria de su antiguo y patriarcal Concejo). Madrid: Instituto de Estudios de Administración Local (España).

Fanjul Peraza, A. (2019). Los astures. Un pueblo céltico del Noroeste Peninsular. Ponferrada: Instituto de Estudios Bercianos. https://ieb.org.es/images/publicaciones/digitales/LosAstures/LosAstures.pdf

Fonte, M., Ranaboldo, C. (2007). Desarrollo rural, territorios e identidades culturales. Perspectivas desde América Latina y la Unión Europea. Opera, 7.

González Rabanal, N. (2005). Importancia de los planes de reordenación en la reconversión del carbón en España. Pecvnia, 1.

Herrero Nogueira, J. (2004). Modo de producción, realidad social e identidad en la minería de Laciana, Tesis de doctorado. España: Universidad Complutense de Madrid, pp. 394-419.

INE (Instituto Nacional de Estadística) (2020). Padrón municipal de habitantes. https:// www.ine.es/nomen2/index.do

Pernas García, J. (2011). Sentencia del TJUE de 24 de junio de 2011, Comisión/Reino de España, asunto C-404/09, Actualidad Jurídica Ambiental, España. http://www.actualidadjuridicaambiental.com/jurisprudencia-al-dia-union-europea-habitats/

Acuerdo Marco para una Transición Justa de la Minería del Carbón y Desarrollo Sostenible de las Comarcas Mineras para el periodo 2019-2027, (2018). Ministerio de Transición Ecológica, Gobierno de España. https://www.irmc.es/Noticias/common/Acuerdo-Marco-para-una-transicion-justa-de-la-mineria-del-carbon-2019.pdf

Ramón, E. (2018): El archivo como institución de la memoria. Dossier Palabra Pública. Chile. https://palabrapublica.uchile.cl/2018/01/11/el-archivo-como-institucion-de-lamemoria/

Rubio Díez, R. y Marcos Herrán, J. (2010). Aproximación al poblamiento castreño en el valle de Laciana. Villablino, León. Zephyrus, 66. 
